\title{
Generalized model of pulse process for dynamic analysis of Sylov's fuzzy cognitive maps
}

\author{
R.A. Isaev ${ }^{1}$, A.G. Podvesovskii ${ }^{1}$ \\ ${ }^{\text {I} B r y a n s k ~ S t a t e ~ T e c h n i c a l ~ U n i v e r s i t y, ~} 50$ let Oktyabrya Blvd. 7, 241035, Bryansk, Russia
}

\begin{abstract}
The article deals with pulse process as a means of dynamic analysis of cognitive models of semi-structured systems. We introduce and substantiate a generalized model of pulse process for Sylov's fuzzy cognitive maps. We offer its implementations for various semantic interpretations of concept influence. The results of experimental validation of the proposed models are presented in the paper.
\end{abstract}

Keywords: cognitive modeling; fuzzy cognitive map; dynamic analysis; pulse process

\section{Introduction}

A cognitive approach is one of the approaches to the study of semi-structured systems, which is widely used at the present time. According to the definition given in [1], this approach focuses on the development of formal models and methods supporting the intelligent problem-solving process as they include human cognitive capabilities (perception, conception, cognition, understanding, explanation) in solving management problems. Structure and target modeling and simulation modeling methods based on cognitive approach are commonly subsumed under the umbrella term "cognitive modeling". In general terms, cognitive modeling refers to the study of structure, functioning and development of a system by analyzing its cognitive model. The cognitive model is based on a cognitive map, which reflects researcher's subjective notion (individual or collective) of the system as a number of semantic categories (known as factors or concepts) and a set of cause-and-effect relations between them.

A cognitive model is an effective tool for exploratory and estimative analysis of the situation. It does not give an opportunity to obtain accurate quantitative characteristics of the system under study, but it allows to assess trends related to its functioning and development, and to identify the key factors influencing these processes. Thus, we can search, generate and develop effective solutions for system management, as well as identify risks and develop strategies to reduce them.

Cognitive modeling starts with creating a cognitive map of the system under study on the basis of information received from experts. The next step includes direct simulation. Its main objectives are forming and testing hypotheses for the structure of the system under study, that can explain its behavior, also developing strategies for various situations in order to reach the specified target states.

Tasks solved by means of cognitive modeling can be divided into two groups:

1. Tasks of structure and target analysis:

- $\quad$ finding the key factors influencing the targets;

- identification of contradictions between the targets;

- identification of feedback loops.

2. Tasks of dynamic analysis (scenario simulation):

- self-development ("what if we do nothing");

- managed development:

○ direct task ("what if");

○ inverse task ("how to").

Thus, the scenario simulation allows prediction of the simulated system states under different control actions, and search for alternative control solutions bringing the system to the target state.

Mathematical apparatus most commonly used to represent cognitive models and underlying the methods for their analysis is fuzzy logic. As a result, there appeared a whole class of cognitive models based on different types of fuzzy cognitive maps (FCM). A detailed overview of such models can be found, for instance, in monograph [3]. One of FCM varieties, well-proven in practical analyzing and modeling of semi-structured organizational, social and economic systems are Sylov's FCMs. They were firstly proposed in [7] and represent the development of signed cognitive maps [6]. For this type of FCMs there was developed quite a wide range of structure and target analysis methods based on the study of such FCM factors as consonance, dissonance and action. A detailed description of these methods can be found in the original monograph [7], and some examples of their application in the study of different organizational and social systems - in papers [2, 4]. The problem of developing and improving methods of Sylov's FCMs dynamic analysis was given far less attention. This article presents an approach to dynamic analysis of this FCM type using a generalized model of pulse process. The proposed approach is based on the notion of pulse process, originally introduced in [6] for the class of signed cognitive maps. We generalize this concept by extending it to the class of FCMs and develop the approach, first mentioned in [5] and described in more detail in monograph [4] (section 3.2). 


\section{Formal definition and structure of Sylov's fuzzy cognitive map}

As previously mentioned, the cognitive model is based on formalization of cause-and-effect relations which occur between factors characterizing the system under study. The result of the formalization represents the system in the form of a cause-andeffect network, termed a cognitive map and having the following form:

$$
G=<E, W>\text {, }
$$

where $E=\left\{e_{1}, e_{2}, \ldots, e_{K}\right\}$ is a set of factors (also called concepts), $W$ is a binary relation on the set $E$, which specifies a set of cause-and-effect relations between its elements.

Concepts can specify both relative (qualitative) characteristics of the system under study, such as popularity, social tension, and absolute, measurable values - population size, cost, etc. Moreover, every concept $e_{i}$ is connected with a state variable $v_{i}$, which specifies the value of the corresponding index at a particular instant. State variables can possess values expressed on a certain scale, within the established limits. Value $v_{i}(t)$ of state variable at instant $t$ is called the state of concept $e_{i}$ at the given instant. Thus, the state of the simulated system at any given instant is described by the state of all concepts included in its cognitive map.

Concepts $e_{i}$ and $e_{j}$ are considered to be connected by relation $W$ (designated as $\left(e_{i}, e_{j}\right) \in W$ or $\left.e_{i} W e_{j}\right)$ if changing the state of concept $e_{i}$ (cause) results in changing the state of concept $e_{j}$ (effect). In this case we say that concept $e_{i}$ influences concept $e_{j}$. Besides, if the value increase of the concept-cause state variable leads to the value increase of the concept-effect state variable, then the influence is considered positive ("strengthening"); if to the decrease - then negative ("inhibition"). Therefore, the relation $W$ can be represented as a union of two disjoint subsets $W=W^{+} \quad W^{-}$, where $W^{+}$is a set of positive relations and $W^{-}$is a set of negative relations.

Fuzzy cognitive model is based on the assumption that the influence between concepts may vary in intensity, whereas, intensity may be constant or variable in time. Taking into account this assumption, $W$ is set as a fuzzy relation, however, its setting depends on the adopted approach to formalization of cause-and-effect relations. A cognitive map with fuzzy relation $W$ is termed a fuzzy cognitive map.

Sylov's fuzzy cognitive map represents FCM, characterized by the following features.

State variables of concepts can possess values on the interval $[0,1]$.

Influence intensity is considered constant, so relation $W$ is specified as a set of numbers $w_{i j}$, characterizing the direction and degree of influence intensity (weight) between concepts $e_{i}$ and $e_{j}$ :

$$
w_{i j}=w\left(e_{i}, e_{j}\right) \text {, }
$$

where $w$ is a normalized index of influence intensity (characteristic function of the relation $W$ ) with the following properties:

a) $-1 \leq w_{i j} \leq 1$;

b) $w_{i j}=0$, if $e_{j}$ does not depend on $e_{i}$ (no influence);

c) $\quad w_{i j}=1$ if positive influence of $e_{i}$ on $e_{j}$ is maximum, i.e. when any changes in the system related to concept $e_{j}$ are univocally determined by the actions associated with concept $e_{i}$;

d) $w_{i j}=-1$ if negative influence is maximum, i.e. when any changes related to concept $e_{j}$ are uniquely constrained by the actions associated with concept $e_{i}$;

e) $w_{i j}$ possesses the value from the interval $(-1,1)$, when there is an intermediate degree of positive or negative influence.

Clearly, FCM of this structure can be graphically represented as a weighted directed graph, which points correspond to elements of set $E$ (concepts) and arcs correspond to nonzero elements of relation $W$ (cause-and-effect relations). Each arc has a weight which is specified by the corresponding value $w_{i j}$. In this case, relation $W$ can be represented as a matrix of dimension $n \times n$ (where $n$ is the number of concepts in the system), which can be considered as the graph adjacency matrix and is termed a cognitive matrix. In addition, each point of the graph also has a weight, which corresponds to the associated concept state and can change over time.

\section{Pulse process as a means of dynamic analysis of cognitive maps}

Dynamic analysis of cognitive maps is based on modeling of concept state dynamics over time. Besides, concept state may change, firstly, due to changes in the state of other concepts influencing this one, and, secondly, due to external actions. We understand external action as a change of the concept state as to the current one under the impact of external factors, i.e. irrespective of the concepts included in the cognitive map. At the same time external actions can be targeted, i.e. they come from the subject performing system control, and untargeted, i.e. due to uncontrollable factors, external to the system. Thus, in the first case we speak about control actions, and in the second case - about disturbing actions (or disturbance).

To describe the dynamics of concept states we use pulse processes. This approach is based on the assumption that changes in the states of all concepts occur at discrete moments of time. State change of concept $e_{i}$ at instant $t$ is called pulse and is denoted by $p_{i}(t)$. Thus,

$$
p_{i}(t)=v_{i}(t)-v_{i}(t-1) \text {. }
$$

It is additionally assumed that influence transmission occurs in one step: changing the state of the concept-cause at instant $t$ results in changing the state of the concept-effect at instant $t+1$.

Let us first give the model of pulse process for signed cognitive maps, i.e. maps which take into account only the directions of influence but not their intensity. For such maps $w_{i j}$ can only take values $-1,0$ or 1 , and the graph arcs are marked with signs "+" and "-“", respectively. The model of pulse process was proposed in [6]: 


$$
p_{i}(t+1)=\sum_{j=1}^{K} \operatorname{sgn}\left(w_{j i}\right) p_{j}(t)
$$

accordingly

$$
v_{i}(t+1)=v_{i}(t)+\sum_{j=1}^{K} \operatorname{sgn}\left(w_{j i}\right) p_{j}(t) .
$$

Thus, the state change (pulse) of each concept in the current step is determined by the pulses of all concepts influencing it and by the ratio of influence signs. Moreover, transmission of positive influence is neutralized by simultaneous transmission of negative influence, and vice versa.

In $[4,5]$, a modified model of pulse process for Sylov's FCM is proposed. The model takes into account both influence transmission between concepts and external actions:

$$
v_{i}(t+1)=\min \left(v_{i}(t)+u_{i}(t+1)+q_{i}(t+1)+\sum_{j=1}^{K} w_{j i} p_{j}(t), 1\right),
$$

where $u_{i}(t+1)$ is a control action on concept $e_{i}$ at instant $t+1 ; q_{i}(t+1)$ is disturbance $e_{i}$ at instant $(t+1)$.

\section{Generalized model of pulse process}

In the framework of Model (1) it is assumed that the state change of concept $e_{j}$ is equal to the difference between its states at the current step and the previous step:

$$
p_{j}(t)=v_{j}(t)-v_{j}(t-1) .
$$

Thus, in dynamic simulation in order to determine the state of dependent concepts we take into account absolute change in states of influencing concepts. This approach is acceptable, but at the same time, it is not the only possible one. In this regard, it is advisable to consider other, alternative approaches to interpreting concept influence and propose alternative models of pulse process on their basis.

However, it is necessary to define a number of requirements to models of pulse process, which must be met by all proposed models in the future, regardless of the assumptions which they are based on.

Firstly, a model of pulse process should unambiguously determine the state of an arbitrary concept $e_{i}$ at instant $(t+1)$, using for this purpose the following available information:

- $\quad$ the state of the same concept $e_{i}$ at instant $t$;

- the states of concepts $e_{j}, \ldots, e_{k}$, influencing concept $e_{i}$, at instant $t$;

- the states of these concepts influencing $e_{i}$, at instant $(t-1)$;

- connection weights (influence intensity) $w_{j i}, \ldots, w_{k i}$ among all influencing concepts and $e_{i}$;

- control and disturbance actions on $e_{i}$ at instant $(t+1)$, if there are any.

Or, more formally:

$$
v_{i}(t+1)=f\left(v_{i}(t), v_{j}(t), \ldots, v_{k}(t), v_{j}(t-1), \ldots, v_{k}(t-1), w_{j i}, \ldots, w_{k i}, u_{i}(t+1), q_{i}(t+1)\right) .
$$

Secondly, the following conditions should be met:

- the values of state variables of concepts belong to the interval $[0,1]$, that is $v_{i}(t+1) \in[0,1]$;

- if influence intensity between concepts $e_{j}$ and $e_{i}$ is equal to 0 , then changing $e_{j}$ state should not cause changing $e_{i}$ state;

- if the states of influencing concepts at the previous step did not change $\left(v_{j}(t)=v_{j}(t-1)\right.$ for all $\left.j\right)$, and there are no control and disturbance actions, then the state of the dependent concept at the current step should not change: $v_{i}(t+1)=v_{i}(t)$;

- when the state of the influencing concept increases (decreases) and the relation is positive, the state of the dependent concept should not decrease (not increase): $v_{i}(t+1) \geq v_{i}(t)$ if $w_{j i}>0$ and $v_{j}(t)>v_{j}(t-1) ; v_{i}(t+1) \leq v_{i}(t)$ if $w_{j i}>0$ and $v_{j}(t)<v_{j}(t-1)$;

- when the state of the influencing concept increases (decreases) and the relation is negative, the state of the dependent concept should not increase (not decrease): $v_{i}(t+1) \leq v_{i}(t)$ if $w_{j i}<0$ and $v_{j}(t)>v_{j}(t-1)$; $v_{i}(t+1) \geq v_{i}(t)$ if $w_{j i}<0$ and $v_{j}(t)<v_{j}(t-1)$;

- a more significant change of the influencing concept with other factors equal should result in a more significant change of the dependent concept: $p_{i}^{1}(t+1) \geq p_{i}^{2}(t+1)$, if $p_{j}^{1}(t) \geq p_{j}^{2}(t)$;

- higher intensity of the influence with other factors equal should result in a more significant change of the dependent concept: $p_{i}^{1}(t+1) \geq p_{i}^{2}(t+1)$, if $w_{j i}^{1} \geq w_{j i}^{2}$.

Let us call the Expression (2) together with the above mentioned conditions a generalized model of pulse process. This model, on the one hand, comprises Model (1) as a possible particular case, and on the other hand, it provides the basis for building other implementations of the pulse process model. 


\section{Implementation of the generalized model of pulse process}

Let us consider alternative implementations of the described generalized model of pulse process, involving different interpretations of concept influence.

\subsection{Pulse process model, based on relative changes of concept states}

Let us assume that concept influence on the system is determined not by the change of its state in general, but by the significance of this change relative to the previous state of this concept. In other words, we consider a relative change of concept states, not an absolute one.

With this view, let us consider pulse $p_{i}(t)$ as a relative state change of concept $e_{i}$ at instant $t$ :

$$
p_{i}(t)=\frac{v_{i}(t)-v_{i}(t-1)}{v_{i}(t-1)} .
$$

Thus, the value of pulse $p_{i}(t)$ shows by what fraction of its state at instant $(t-1)$ concept $e_{i}$ has changed.

Now, let us define the way of influence transmission between directly related concepts. Suppose there is a relation between concepts $e_{j}$ and $e_{i}$, whose strength is equal to $w_{j i}$. To begin with, knowing $p_{j}(t)$ - the relative change of state $e_{j}$ at instant $t$, let us define the relative change of state $e_{j}$ at instant $(t+1)$.

It is necessary to consider the conditions of the generalized model, and the following additional conditions:

- if $p_{j}(t)=0$ or $w_{j i}=0$, then $p_{i}(t+1)=0$;

- if $w_{j i}=1$, then $p_{i}(t+1)=p_{j}(t)$.

The following operation satisfies these conditions:

$$
p_{i}(t+1)=w_{j i} p_{j}(t)
$$

Finally, let us define the state of concept $e_{j}$ at instant $(t+1)$. Note that

So,

$$
p_{i}(t+1)=\frac{v_{i}(t+1)-v_{i}(t)}{v_{i}(t)} .
$$

$$
v_{i}(t+1)=v_{i}(t)+v_{i}(t) w_{j i} p_{j}(t)
$$

The resulting model can be easily generalized in the case of multiple influencing concepts:

$$
v_{i}(t+1)=v_{i}(t)+v_{i}(t) \sum_{j=1}^{K} w_{j i} p_{j}(t) .
$$

As one of the conditions of the generalized model is that the concept states range within the interval $[0,1]$, then we should add the following constraints to the model:

$$
v_{i}(t+1)=\max \left(\min \left(v_{i}(t)+v_{i}(t) \sum_{j=1}^{K} w_{j i} p_{j}(t), 1\right), 0\right) .
$$

Moreover, control and disturbance actions on $e_{i}$ should also be defined in terms of relative changes. For example, the control action $u_{i}(t+1)=0,1$ means "to increase the value of $i$-concept state variable by $10 \%$ of its current value".

Thus, we obtain the final version of the model:

$$
v_{i}(t+1)=\max \left(\min \left(v_{i}(t)+v_{i}(t) u_{i}(t+1)+v_{i}(t) q_{i}(t+1)+v_{i}(t) \sum_{j=1}^{K} w_{j i} p_{j}(t), 1\right), 0\right) .
$$

\subsection{Multiplicative model of pulse process}

Let us consider another model, which also takes into account relative changes of concept states but implies a slightly different interpretation of these changes. This model is not equivalent to that described above, but they both proceed from similar prerequisites.

In this case, relative change of concept $e_{j}$ state shows what fold this concept changed at instant $t$ compared with its state at instant $(t-1)$ :

$$
p_{i}(t)=\frac{v_{i}(t)}{v_{i}(t-1)} .
$$

Let us define the way of influence transmission between directly related concepts In this case, the following conditions should be taken into account in addition to those of the generalized model:

- if $w_{j i}=1$, then $p_{i}(t+1)=p_{j}(t)$;

- if $w_{j i}=0$ or $p_{j}(t)=1$, then $p_{i}(t+1)=1$; 
- if $w_{j i}=-1$, then $p_{i}(t+1)=\frac{1}{p_{j}(t)}$.

Exponential operation satisfies these conditions:

$$
p_{i}(t+1)=\left(p_{j}(t)\right)^{w_{j i}}
$$

Now we can easily determine the state of concept $e_{i}$ at instant $(t+1)$ :

$$
v_{i}(t+1)=v_{i}(t)\left(p_{j}(t)\right)^{w_{j i}}
$$

Generalization of the model in case of multiple influencing concepts is the following:

$$
v_{i}(t+1)=v_{i}(t) \prod_{j=1}^{K}\left(p_{j}(t)\right)^{w_{j i}} .
$$

This model does not operate on negative values (excluding connection weights used as exponents). This guarantees the fulfillment of the condition $v_{i}(t+1) \geq 0$. To fulfill the other condition of the generalized model, namely $v_{i}(t+1) \leq 1$, let us add the constraint:

$$
v_{i}(t+1)=\min \left(v_{i}(t) \prod_{j=1}^{K}\left(p_{j}(t)\right)^{w_{j i}}, 1\right) .
$$

Control and disturbance actions within this model should be specified on the basis of the interpretation "concept state has changed $n$-fold". For example, the control action $u_{i}(t+1)=2$ means "to double the concept state as compared to its current state".

Thus, the final version of the model under study is:

$$
v_{i}(t+1)=\min \left(v_{i}(t) u_{i}(t+1) q_{i}(t+1) \prod_{j=1}^{K}\left(p_{j}(t)\right)^{w_{j i}}, 1\right) .
$$

\section{Experimental validation of the discussed pulse process models}

For experimental validation and comparison of the examined models, let us perform dynamic analysis of a cognitive map using each of them, with the same initial data.

Fig. 1 shows a fragment of the cognitive map used for the experiment. Connection weights are assigned the following values: $w_{12}=0,9 ; w_{23}=-0,8 ; w_{31}=0,7$. The initial concept states are specified as: $v_{1}(1)=0,2 ; v_{2}(1)=0,3 ; v_{3}(1)=0,8$.

Suppose there is a control action on concept 1 , which results in its transition to a state $v_{1}(2)=0,6$. Influenced by the initial pulse, concept states begin to change in accordance with the rules defined by each model of pulse process.

Fig. 2-4 give graphs of concept state changes during the operation of three models of pulse process. The horizontal axis measures simulation steps; the vertical axis measures the state of the corresponding concept. For the graphs we use the following notations:

- "Model 1" - the results obtained using the additive model (1);

- "Model 2" - the results obtained using the additive model (3) based on relative changes of concept states;

- "Model 3" - the results obtained using the multiplicative model (4) based on relative changes of concept states.

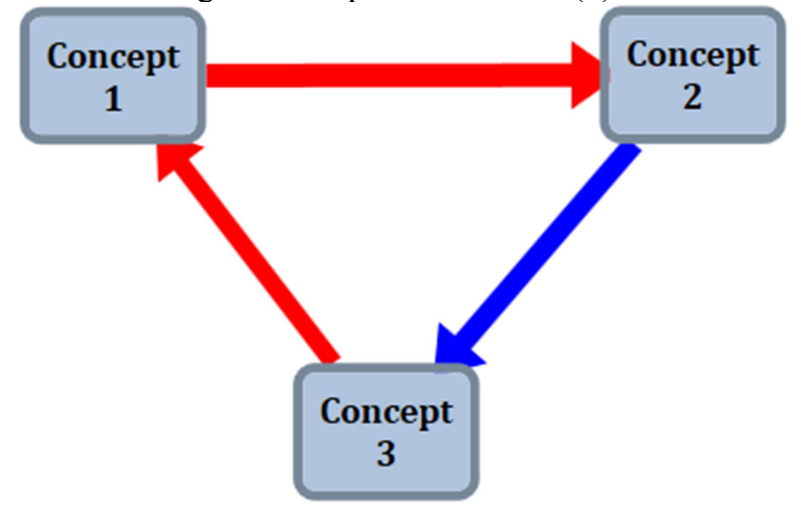

Fig. 1. Fragment of a fuzzy cognitive map used for the experiment.

Of principal interest for interpretation is influence transmission between directly related concepts, differently occurring within different models, eventually providing different results. Thus, in models 2 and 3 , implying relative change of concept states, the state of the second concept at the 3rd simulation step increased more than in model 1. Similarly, account taken of relative changes results in more significant decrease in the state of the third concept at the 4th step. Similar regularities are typical for the subsequent steps. 


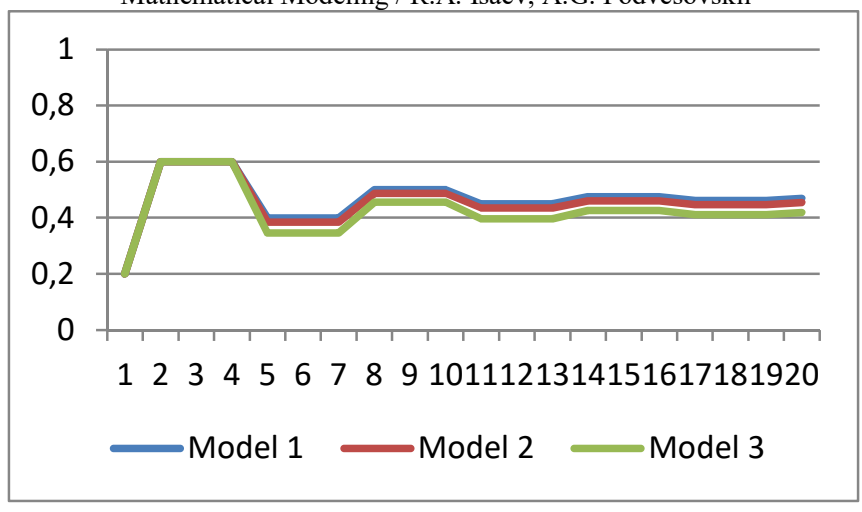

Fig. 2. Dynamics of state change of concept 1 .

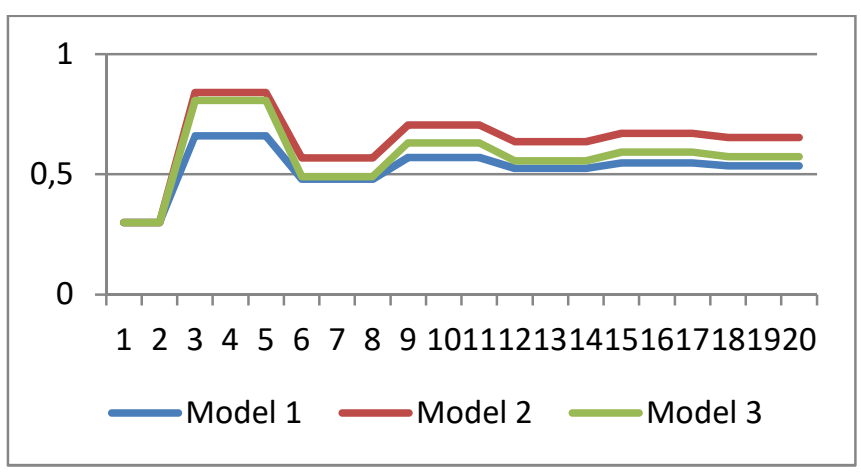

Fig. 3. Dynamics of state change of concept 2 .

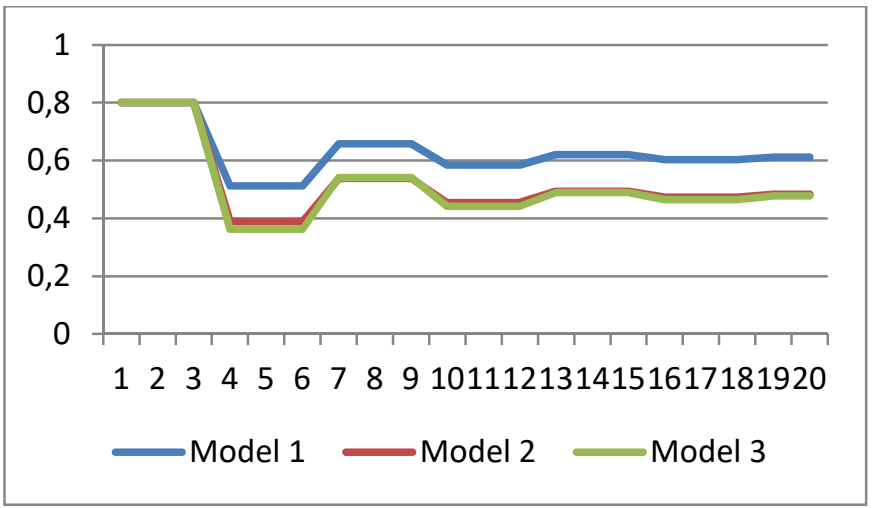

Fig. 4. Dynamics of state change of concept 3 .

Describing the results in general, it should be noted that:

- all models operate correctly regarding the influence transmission: the directions of concept state changes correspond to the signs of influences;

- $\quad$ all models are stable: pulse decays with time, which results in the system transition to some stable state;

- as a result of simulation the state of each concept has changed in the same direction for all models (the states of the first and second concepts increased, the state of the third one decreased in comparison with the initial one). These results are generally consistent with the intuitive notion of the system changes pattern, which also validates the models;

- differences in predictions obtained by different models are quite well explained by the underlying prerequisites (concerning the nature of influences among concepts).

\section{Conclusion}

The paper introduces a generalized model of pulse process for Sylov's fuzzy cognitive maps. This model, on the one hand, represents a generalization of previously developed models, and on the other hand, can serve as a basis for building other variations of the pulse process model.

Also, the proposed alternative implementations of the described generalized pulse process model provide diverse interpretations of concept influence. Experimental validation of these implementations has been carried out confirming their correctness and operability.

Among the possible directions for further research, the following are of major interest: 
- identifying characteristics and making requirements to the methods of expert identification of FCM parameters in different pulse process models;

- identifying characteristics and making requirements to the methods of identification of FCM parameters on the basis of statistical data in different pulse process models;

- developing methods for selecting an optimal pulse process model based on the analysis of available statistical and expert data.

\section{References}

[1] Avdeeva ZK, Kovriga SV, Makarenko DI. Cognitive Modeling Approach to Control of Semi-Structured Systems (Situations)ю Managing Large Systems 2007; 16: 26-39. (in Russian)

[2] Averchenkov VI, Kozhukhar VM, Podvesovskii AG, Sazonova AS. Monitoring and Prediction of Regional Demand for Highest Scientific Degree Specialists: monograph. Edited by Averchenkov VI, Kozhukhar VM. Bryansk: Bryansk State Technical University Press, 2010; 163 p. (in Russian)

[3] Borisov VV, Kruglov VV, Fedulov AS. Fuzzy Models and Networks. Moscow: "Goryachaya Liniya - Telekom" Publisher, 2012; 284 p. (in Russian)

[4] Erokhin DV, Lagerev DG, Laricheva EA, Podvesovskii AG. Strategic Enterprise Innovation Managemnet: monograph. Bryansk: Bryansk State Technical University Press, 2010; 196 p. (in Russian)

[5] Podvesovskii AG, Lagerev DG, Korostelyov DA. Application of Fuzzy Cognitive Models for Construction of Alternatives Set in Decision Problems. Bulletin of Bryansk State Technical University 2009; 4(24): 77-84. (in Russian)

[6] Roberts FS. Discrete Mathematical Models with Application to Social, Biological and Environmental Problems. Prentice-Hall, Englewood Cliffs, 1976.

[7] Sylov VB. Strategic Decision Making in Fuzzy Environment. Moscow: “INPRO-RES” Publisher, 1995; 228 p. (in Russian) 